\title{
EFEITO DA CONCENTRAÇÃO DE ADITIVO DE CARBONO EM FORMA DE GRAFITE NA MACROPOROSIDADE, DENSIDADE APARENTE E MORFOLOGIA DE PLACAS DE BATERIA CHUMBO- ÁCIDO
}

\section{EFFECT OF THE CONCENTRATION OF CARBON ADDITIVE IN GRAPHITE FORM IN THE MACROPOROSITY, APPARENT DENSITY AND MORPHOLOGY OF LEAD-ACID BATTERY PLATES}

\author{
G. B. FEIJÓ ${ }^{1}$, M. de S. L. FARIA ${ }^{1}$, L. C. SANTANA ${ }^{1,{ }^{*}}$, C. M. de CARVALHO ${ }^{2}$ e G. A. O. BRITO ${ }^{3}$
}

${ }^{1}$ Federal University of Espírito Santo, Department of Rural Engeneering, Alegre, Espírito Santo, Brazil

${ }^{2}$ Federal University of Espírito Santo, Postgraduate Program in Chemical Engineering, Alegre, Espírito Santo, Brazil

${ }^{3}$ Federal University of Uberlândia, Department of Chemistry, Uberlândia, Minas Gerais, Brazil

${ }^{*}$ Corresponding author. Federal University of Espírito Santo, Department of Rural Engineering, Alegre, Espírito Santo, Brazil, Phone: +5528998816761

e-mail addressl: lucascarvalhosantana@outlook.com.br (L. C. Santana).

\begin{tabular}{l} 
A R T I C L E I N F O \\
\hline Article history: \\
Received 2020-06-30 \\
Accepted 2020-10-20 \\
Available online 2020-10-20 \\
pa lavras-chave \\
Baterias de chumbo-ácido \\
Aditivo de Carbono \\
Morfologia \\
keywords \\
Lead Acid Batteries \\
Carbon Additive \\
Morphology \\
\end{tabular}

A B S T R A C T

Batteries are the most widely used energy storage devices. Thus, different alternatives have been studied as a way to improve the properties of these systems. The insertion of additives in the composition of the active material of lead-acid battery plates, has been considered as one of the alternatives to promote such improvement. Thus, the present work proposes to evaluate the influence of the insertion of carbon additives in the form of graphite on the morphology of lead-acid battery plates. In view of the results obtained, it is concluded that the macroporosity has an inversely proportional relationship with the apparent density and that the morphology and color of the material's surface are drastically modified with the increase of the graphite content.

R E S U M O

As pilhas e baterias são os dispositivos de acumulação de energia mais utilizados. Assim, temse estudado diferentes alternativas como forma de melhorar propriedades desses sistemas. A inserção de aditivos na composição do material ativo das placas de baterias chumbo-ácido, tem sido conceituada como uma das alternativas para promover tal melhora. Desse modo,o presente trabalho se propõe a avaliar a influência da inserção de aditivos de carbono na forma de grafite na morfologia das placas de baterias chumbo-ácido. Tendo em vista os resultados obtidos, conclui-se que a macroporosidade possui uma relação inversamente proporcional com a densidade aparente e que a morfologia e cor da superfície do material são drasticamente modificados com o aumento do conteúdo de grafite. 


\section{INTRODUÇÃO}

Para suprir a demanda energética atual, bem como a necessidade de utilização de fontes de energia alternativas, os sistemas de armazenamento de energia surgem como uma solução. Os acumuladores de energia de chumbo-ácido possuem grande destaque na indústria automotiva, sendo este setor o de maior aplicabilidade das mesmas (SBPE, 2017).

Pesquisas na área de carros elétricos fomentam a inserção de aditivos no material ativo negativo da bateria como forma de melhorar a eficiência de aceitação de carga e aumentar a durabilidade, principalmente quando submetida a um estado parcial de carga. $\mathrm{O}$ destaque é para os aditivos à base de carbono implantados nas placas negativas das baterias chumboácido (TSURUMAKI, 2015; PAVLOV, 2011).

Diante disso, o presente trabalho objetivou analisar mudanças físicas (na morfologia, densidade aparente e macroporosidade) ocorridas no material precursor negativo devido à adição de grafite Micrograf 9930MA nesse.

\section{METODOLOGIA}

\subsection{Confecção, Empaste e Cura das Miniplacas}

Foram confeccionadas 30 minigrades para o lote sem aditivo e 10 minigrades para cada uma das diferentes concentrações de aditivos $(0,50 \%, 0,75 \%, 1 \%$ e $2 \%)$. As dimensões destas minigrades eram de $3,8 \mathrm{~cm}$ x $1,6 \mathrm{~cm}$.

Os materiais sólidos (grafite e óxido de chumbo) foram previamente misturados a seco, e após, foi adicionada água destilada gradualmente à mistura, contida em um almofariz de porcelana. A mistura foi realizada por um pistilo de porcelana, à temperatura ambiente.

Confeccionada a pasta, foi realizado o processo de empaste, onde aquela foi aplicada sobre as minigrades. Para isso, as minigrades foram posicionadas sobre uma superfície plana recoberta por papel pardo umedecido com água destilada.

Após o empaste, as miniplacas foram colocadas em uma câmara de cura, onde permaneceram por três dias. Embora não houvesse dispositivos de controle de temperatura e umidade, a configuração da câmara de cura foi projetada de modo que esses parâmetros oscilassem o menos possível. Dessa forma, a câmara permaneceu selada, revestida com papel jornal e com um baixo nível de água em seu interior. Ao final dessa etapa, a pasta passa a ser denominada material precursor e a miniplaca (minigrade empastada) passa a ser denominada miniplaca precursora.

\subsection{Teste de Macroporosidade}

Consiste em mergulhar três placas de cada lote, selecionadas de modo aleatório, em um recipiente com água destilada, em quantidade suficiente para imergir toda a miniplaca. O tempo de mergulho das miniplacas empastadas no recipiente é de 30 minutos e, após, mede-se a massa de cada miniplaca, durante 35 minutos, em intervalos de 5 minutos. A Equação (1) determina o modo de se obter a macroporosidade do material (WILSON, 1999).

$\%$ porosidade $=\frac{V_{\text {água }}}{V_{\text {água }}+V_{\text {chumbo }}} \times 100 \%$

A partir dos dados advindos da Equação (1), elabora-se um gráfico que relaciona a perda de massa da miniplaca em função do tempo decorrido durante a pesagem da miniplaca, após sua imersão em água destilada. O gráfico possui uma região linear, que se inicia entre 4 e $7 \mathrm{~min}$. Essa região é estendida até o ponto de zero segundo e assim, é obtida a massa da miniplaca saturada de água no ponto zero segundo.

A medida da densidade aparente ( $\rho$ ap) leva em consideração a fase sólida do material precursor juntamente com os seus poros. A densidade aparente do material precursor é dada pela Equação (2), sendo mps, a massa da placa seca; Va, o volume de água na miniplaca e $\mathrm{VpbO}$, o volume de óxido de chumbo na miniplaca.

$\rho=\frac{m_{p s}}{V_{P b}+V_{a}}$

Na Equação (2), Va é encontrado utilizando-se a massa de água (diferença entre miniplaca molhada em zero segundo, massa da miniplaca seca após o dessecador e massa da minigrade) e a densidade da água $\left(1 \mathrm{~g} / \mathrm{cm}^{3}\right)$. Assim como $\mathrm{Vpb}$ pode ser encontrado pela divisão da massa de $\mathrm{PbO}$ (diferença entre miniplaca seca após dessecador e massa da minigrade) pela densidade do $\mathrm{PbO}\left(9,35 \mathrm{~g} / \mathrm{cm}^{3}\right)$.

\subsection{Microscopia ótica}

Para os testes de microscopia ótica, utilizou-se o microscópio óptico digital da marca Hirox modelo KH-7700, que dispõe de uma fonte luminosa, câmera digital, monitor e software. A ampliação foi de 400 vezes em relação ao tamanho das amostras. Para essa análise, observaram-se miniplacas representativas de cada lote.

\section{RESULTADOS E DISCUSSÃO}

\subsection{Testes de Macroporosidade e Densidade Aparente}

As Figuras 1 e 2 mostram as macroporosidades e densidades médias em relação ao conteúdo de aditivo adicionado à pasta. 


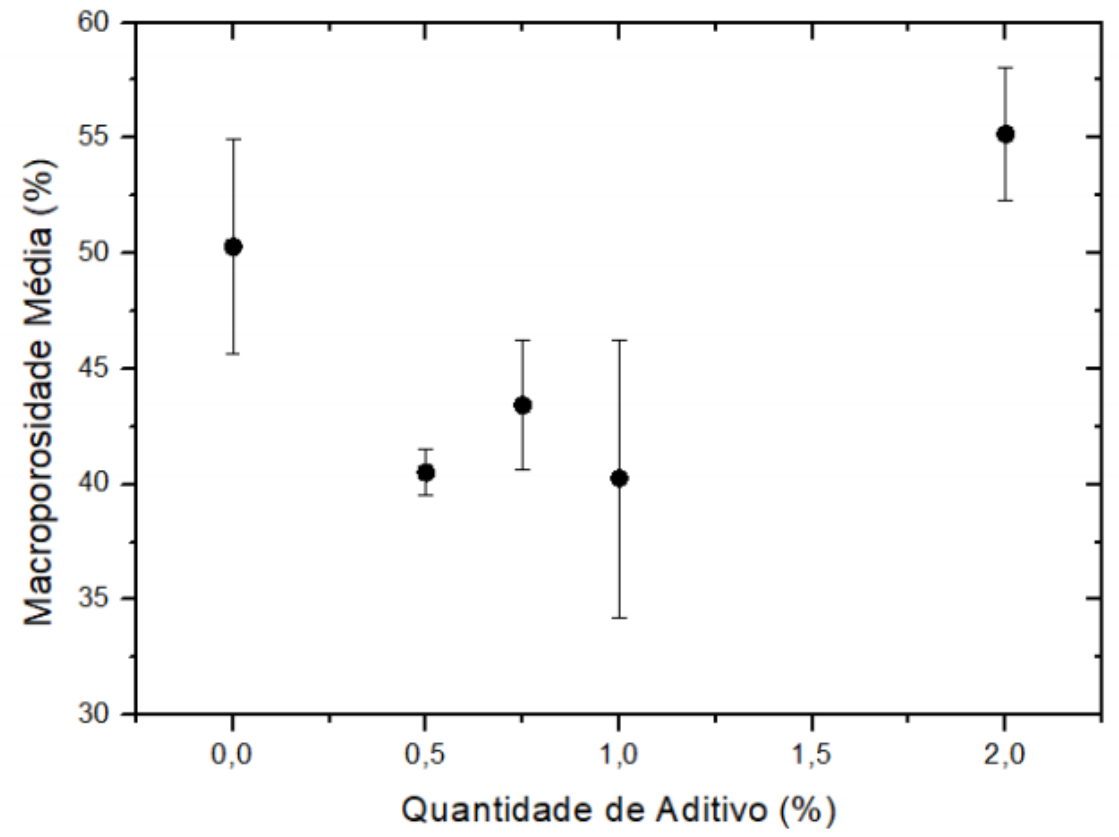

Figure 1 - Relação da macroporosidade média com cada conteúdo de aditivo. (Fonte: Os autores).

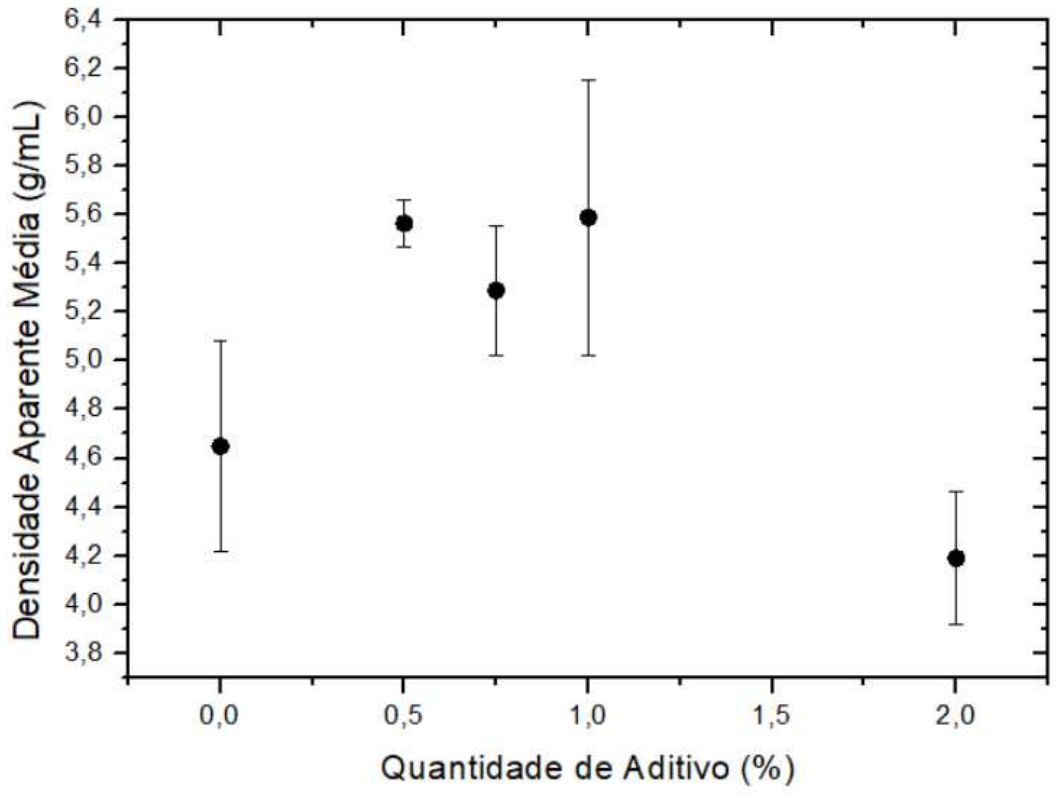

Figure 2 - Relação da densidade aparente média com cada conteúdo de aditivo. (Fonte: Os autores).

Da Figura 1, observa-se que a macroporosidade do material diminui nas concentrações 0,$5 ; 0,75$ e $1 \%$ de aditivo, enquanto aumenta na concentração de $2 \%$ em relação ao material sem aditivo. Na figura 2 , a densidade aparente média aumenta nas concentrações de 0,$5 ; 0,75$ e $1 \%$, enquanto diminui na concentração de $2 \%$ de aditivo em relação ao material sem aditivo.
Em um primeiro momento, pode parecer incongruente que a densidade aparente média aumente com o aumento de aditivo, já que a densidade do grafite é muito menor do que a densidade do $\mathrm{PbO}$, poder-se-ia esperar que quanto maior a quantidade de grafite no material, menos denso esse se tornaria (TSURUMAKI, 2015). 


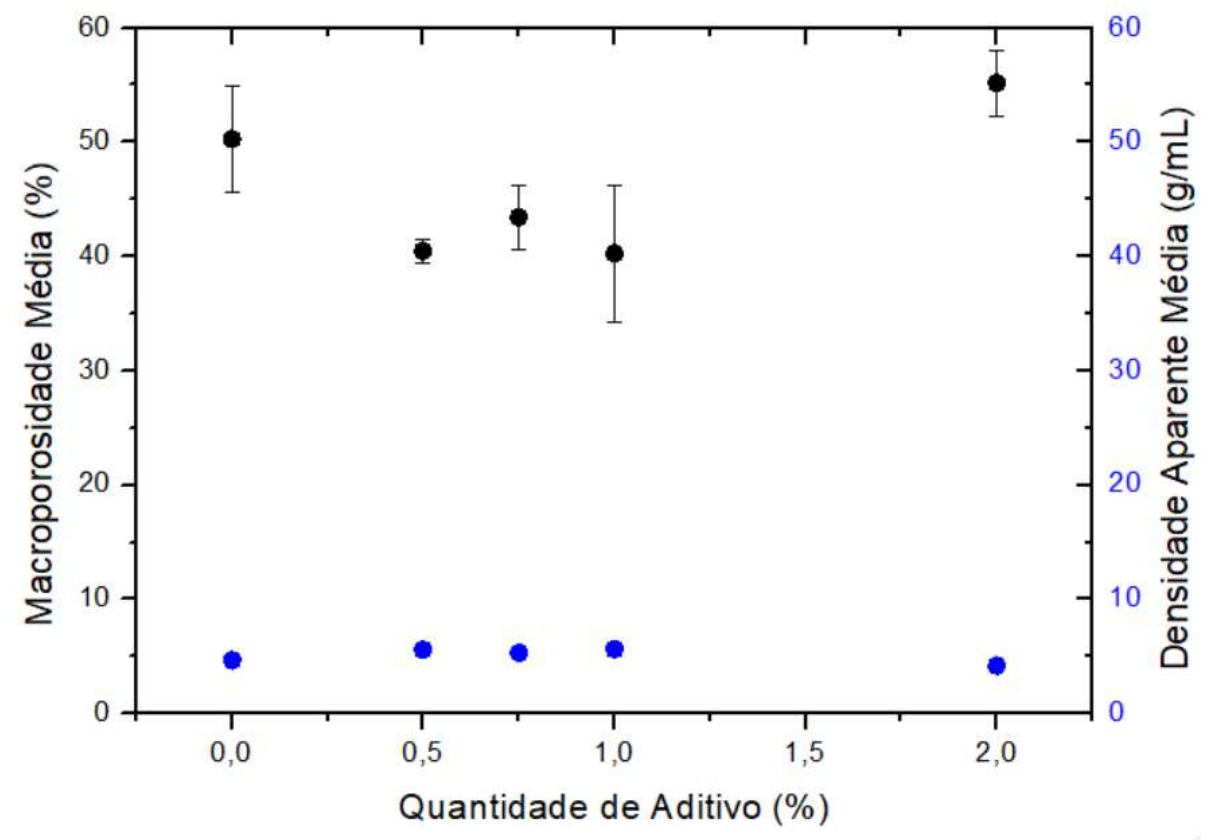

Figure 3 - Gráficos sobrepostos de macroporosidade média e densidade aparente com cada conteúdo de aditivo. (Fonte: Os autores)

No entanto, da Figura 3 (as barras de erro nos pontos de densidade aparente média não aparecem porque se encontram em uma escala muito menor em relação à escala da macroporosidade média), com as curvas de macroporosidade média e densidade aparente média sobrepostas, fica evidente que a densidade aparente possui uma relação inversamente proporcional à macroporosidade e esse resultado está em acordo com H. Bode (1977). Além disso, no próprio trabalho de Tsurumaki, 2015, há exemplo de densidade aparente de material precursor negativo com aditivo de grafite de $0,2 \%$ de massa aumentando em relação ao material sem aditivo. Assim, as respostas para esse fenômeno devem ser melhor investigadas.

\subsection{Testes de Macroporosidade e Densidade Aparente}

As Figuras 4-8 mostram os aspectos físicos das superfícies de miniplacas sem e com aditivo. Da Figura 4 é possível observar que a superfície do material precursor sem aditivo possui a cor característica amarronzada, com alguns macroporos (pontos mais escuros) visíveis à microscopia ótica.

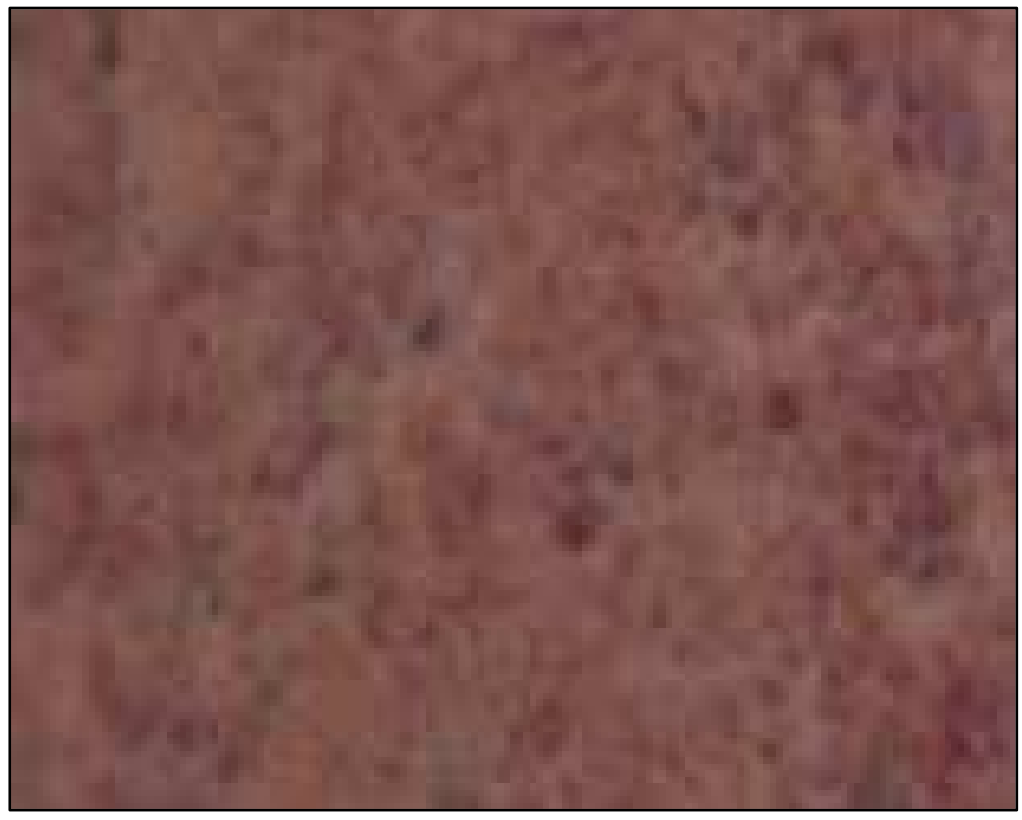

Figure 4 - Aspecto físico das superfícies de uma miniplaca sem aditivo. (Fonte: Os autores) 
Com $0,5 \%$ de aditivo na pasta (Figura 5), a cor do material precursor negativo muda de totalmente amarronzada para um amarronzado mais claro com pontos acinzentados claros e é possível observar grãos de grafite na superfície do material.

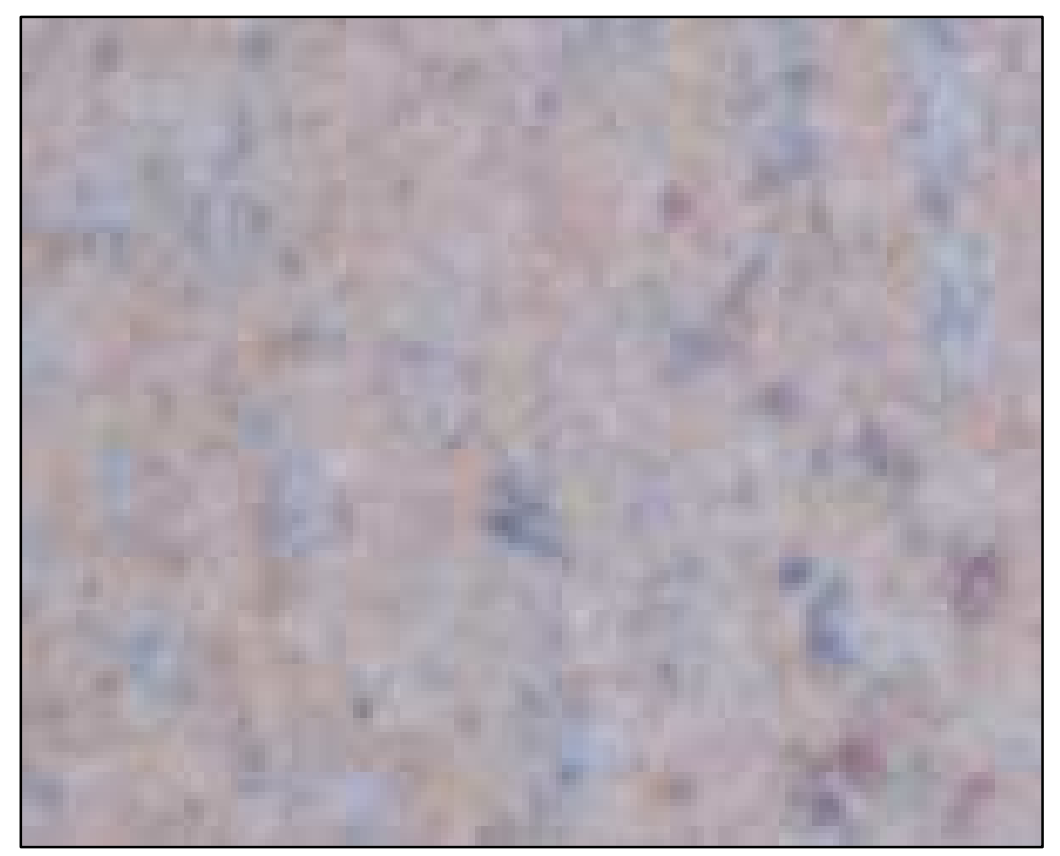

Figure 5 - Aspecto físico das superfícies de uma miniplaca com $0,5 \%$ de aditivo (Fonte: Os autores)

Esses grãos vão se tornando maiores e mais perceptíveis quanto maior a quantidade de aditivo (Figuras 6, 7 e 8). Nessas últimas figuras, a superfície do material possui cor predominantemente acinzentada. É possível observar também que os grãos de grafite ficam homogeneamente espalhados pelo material precursor em todas as concentrações.

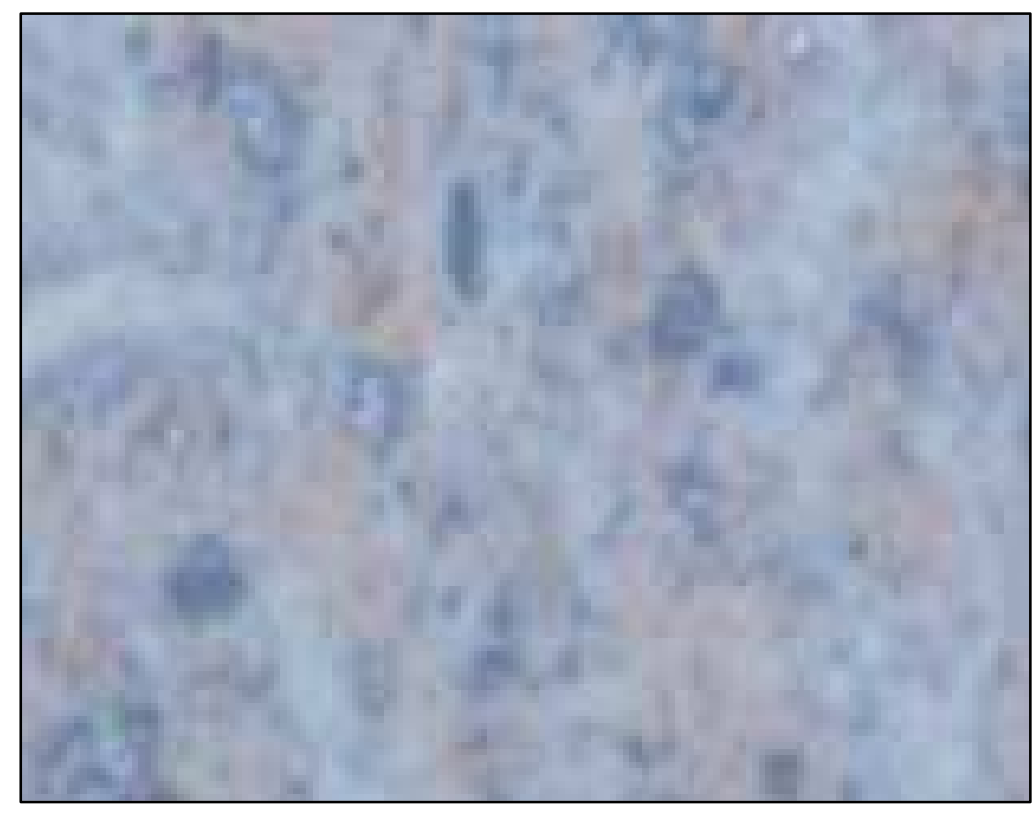

Figure 6 - Aspecto físico das superfícies de uma miniplaca com $0.75 \%$ de aditivo. (Fonte: Os autores) 


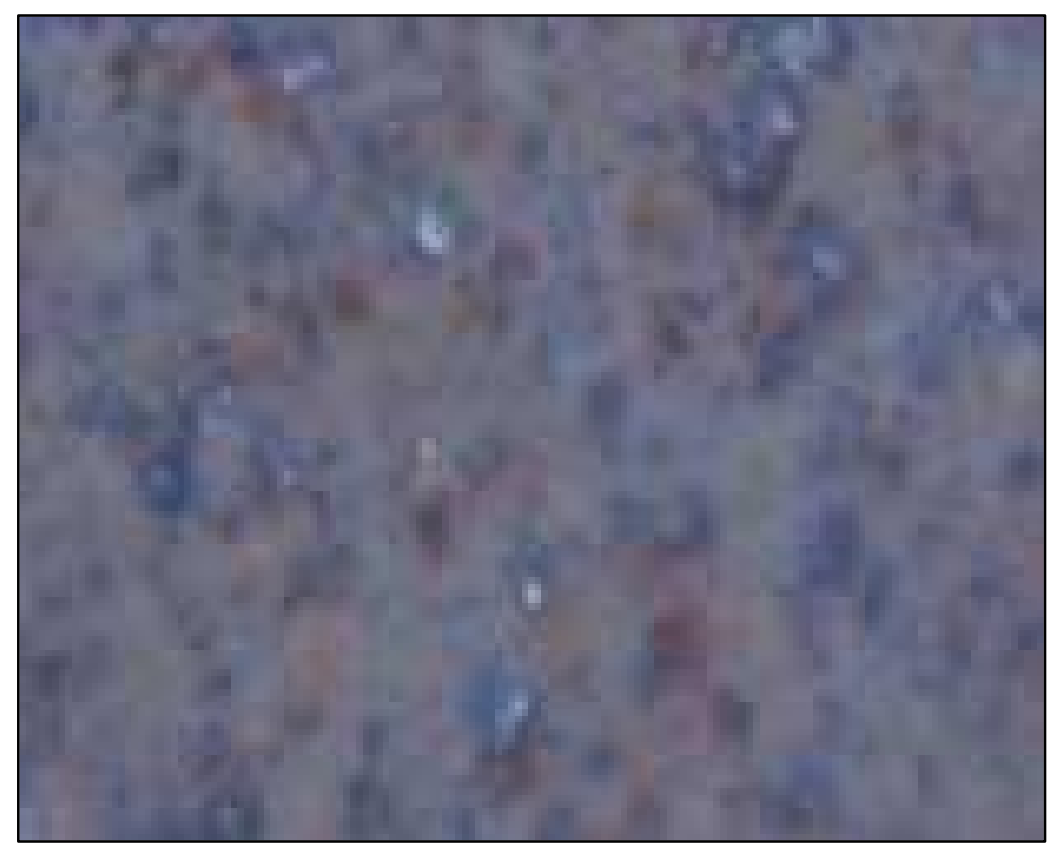

Figure 7 - Aspecto físico das superfícies de uma miniplaca com 1\% de aditivo. (Fonte: Os autores)

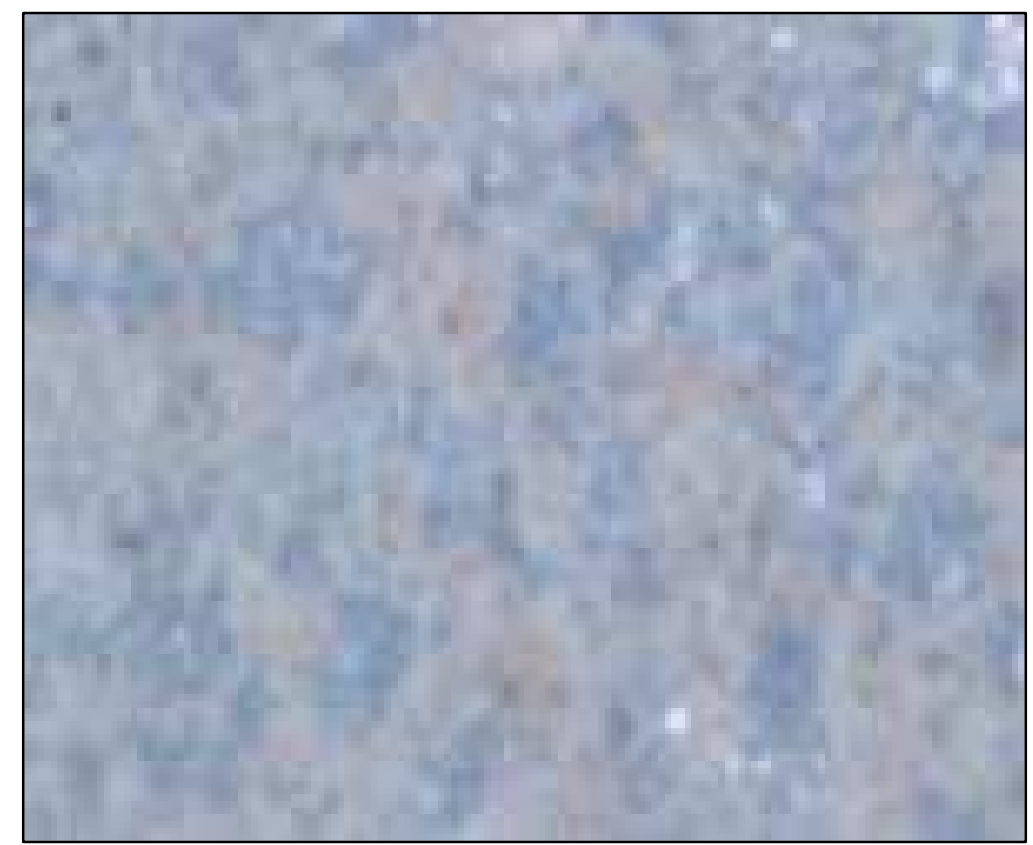

Figure 8 - Aspecto físico das superfícies de uma miniplaca com $2 \%$ de aditivo. (Fonte: Os autores)

\section{CONCLUSÃO}

Conclui-se que a macroporosidade possui uma relação inversamente proporcional com a densidade aparente do material precursor negativo e que a maior quantidade de aditivo, com uma densidade menor do que o $\mathrm{PbO}$, não necessariamente implicará em menor densidade aparente. Aspectos físicos, como morfologia e cor da superfície do material precursor, são drasticamente mudados com o aumento do conteúdo de grafite, onde é possível observar que os grãos desse se espalham homogeneamente por toda a superfície do material precursor.

\section{REFER ÊNCIAS}

BODE, H. Lead-Acid Batteries, John Wiley \& Sons, N.Y. (1977).

PAVLOV, D. Lead-Acid Batteries: Science and Technology. Elsevier Science, 2011. $1^{\mathrm{a}}$ ed.

SOCIEDADE BRASILEIRA DE PLANEJAMENTO ENERGÉTICO, SBPE. Sistemas de Armazenamento de Energia em Redes Inteligentes: Características, Oportunidades e Barreiras. Disponível em : $<$ http://new.sbpe.org.br/artigo/sistemas-

dearmazenamento-de-energia-eletrica-em-redes- 
inteligentes-caracteristicas-oportunidadese-barreiras/>. Acesso em: 24 set. 2017.

TSURUMAKI, M. Carbono no Material Ativo de Baterias Chumbo-Ácido. 2015. 116 f. Dissertação (Mestrado em Química) - Universidade Federal de São Carlos, São
Carlos, 2015.

WILSON, M. A.; CARTER, M. A.; HOFF, W. D. British Standard and RILEM water absorption tests: Acritical evaluation. Materials and Structures, Vol. 32, p. 571$578,1999$. 